\title{
Targeting FLT3 mutations in AML: review of current knowledge and evidence
}

\author{
Naval Daver ${ }^{1} \cdot$ Richard F. Schlenk ${ }^{2} \cdot$ Nigel H. Russell $^{3} \cdot$ Mark J. Levis $^{4}$ \\ Received: 29 August 2018 / Revised: 18 October 2018 / Accepted: 24 October 2018 / Published online: 16 January 2019 \\ (c) The Author(s) 2019. This article is published with open access
}

\begin{abstract}
Genomic investigations of acute myeloid leukemia (AML) have demonstrated that several genes are recurrently mutated, leading to new genomic classifications, predictive biomarkers, and new therapeutic targets. Mutations of the FMS-like tyrosine kinase 3 (FLT3) gene occur in approximately $30 \%$ of all AML cases, with the internal tandem duplication (ITD) representing the most common type of FLT3 mutation (FLT3-ITD; approximately 25\% of all AML cases). FLT3-ITD is a common driver mutation that presents with a high leukemic burden and confers a poor prognosis in patients with AML. The prognostic value of a FLT3 mutation in the tyrosine kinase domain (FLT3-TKD), which has a lower incidence in AML (approximately $7-10 \%$ of all cases), is uncertain. Accumulating evidence demonstrates that FLT3 mutational status evolves throughout the disease continuum. This so-called clonal evolution, together with the identification of FLT3-ITD as a negative prognostic marker, serves to highlight the importance of FLT3-ITD testing at diagnosis and again at relapse. Earlier identification of FLT3 mutations will help provide a better understanding of the patient's disease and enable targeted treatment that may help patients achieve longer and more durable remissions. First-generation FLT3 inhibitors developed for clinical use are broad-spectrum, multikinase inhibitors; however, next-generation FLT3 inhibitors are more specific, more potent, and have fewer toxicities associated with off-target effects. Primary and secondary acquired resistance to FLT3 inhibitors remains a challenge and provides a rationale for combining FLT3 inhibitors with other therapies, both conventional and investigational. This review focuses on the pathological and prognostic role of FLT3 mutations in AML, clinical classification of the disease, recent progress with next-generation FLT3 inhibitors, and mechanisms of resistance to FLT3 inhibitors.
\end{abstract}

\section{AML genetic landscape: risk categorization and recommendations for FLT3 testing}

Acute myeloid leukemia (AML) is a malignancy of proliferative, clonal, abnormally, or poorly differentiated cells of the hematopoietic system, characterized by clonal

Mark J. Levis

levisma@jhmi.edu

1 Department of Leukemia, The University of Texas MD Anderson Cancer Center, Houston, TX, USA

2 National Center of Tumor Diseases, German Cancer Research Center, Heidelberg, Germany

3 Centre for Clinical Haematology, Nottingham University Hospitals NHS Trust, Nottingham, UK

4 Sidney Kimmel Comprehensive Cancer Center, Johns Hopkins University, Baltimore, MD, USA evolution and genetic heterogeneity [1, 2]. Genetic alterations are recurrent and include amplifications, deletions, rearrangements, and point mutations [2, 3]. Because cytogenetic profiles are important prognostic indicators of clinical outcomes, characterizing chromosomal abnormalities in AML helps stratify patients according to risk and guide therapeutic decisions.

Prognostic risk is defined at diagnosis based on the presence of certain cytogenetic and molecular aberrations [4-7]. Guidelines for AML classification and risk stratification have been established by several organizations, including the World Health Organization (WHO), National Comprehensive Cancer Network (NCCN), and European LeukemiaNet (ELN) [4, 5]. Although the WHO lists FMSlike tyrosine kinase 3 internal tandem duplication (FLT3ITD) as a molecular genetic alteration significantly affecting the clinical outcome in patients with AML in specific cytogenetic subgroups [7], it does not group FLT3 mutations into a single category but rather divides them into 
Table 1 NCCN 2017 AML risk stratification based on validated cytogenetics and molecular abnormalities [5]

\begin{tabular}{lll}
\hline Risk status & Cytogenetics & Molecular abnormalities \\
\hline Favorable risk & $\begin{array}{l}\text { Core binding factor: inv(16) or } \\
\mathrm{t}(16 ; 16) \text { or } \mathrm{t}(8 ; 21) \text { or } \mathrm{t}(15 ; 17)\end{array}$ & $\begin{array}{l}\text { Normal cytogenetics: } \\
\text { NPM1 mutation in the absence of } \text { FLT3-ITD or } \\
\text { isolated biallelic (double) CEBPA mutation } \\
\text { Core binding factor with } \text { KIT mutation }\end{array}$ \\
Intermediate risk & $\begin{array}{l}\text { Normal cytogenetics: } \\
+8 \text { alone }\end{array}$ & \\
& $\mathrm{t}(9 ; 11)$ & \\
& Other nondefined & Normal cytogenetics: \\
Poor risk & Complex $(\geq 3$ clonal chromosomal & With FLT3-ITD mutation \\
& abnormalities): & \\
& Monosomal karyotype & \\
& $-5,5 \mathrm{q}-,-7,7 \mathrm{q}-$ & \\
& $11 \mathrm{q} 23-$ non $\mathrm{t}(9 ; 11)$ & \\
& $\mathrm{inv}(3), \mathrm{t}(3 ; 3)$ & \\
$\mathrm{t}(6 ; 9)$ & \\
$\mathrm{t}(9 ; 22)$ & \\
\hline
\end{tabular}

$A M L$ acute myeloid leukemia, CEBPA CCAAT/enhancer-binding protein alpha, FLT3 FMS-like tyrosine kinase 3, ITD internal tandem duplication, NCCN National Comprehensive Cancer Network, NPM1 nucleophosmin many subgroups. Thus, the focus of this review will be on the latter two sets of guidelines, NCCN and ELN.

The NCCN and ELN guidelines (Tables 1 and 2) stratify patients into three risk groups: favorable, intermediate, and poor/adverse. The NCCN Clinical Practice Guidelines in Oncology classify patients with AML with normal cytogenetics harboring the FLT3-ITD or TP53 mutations as poor risk. Additionally, because FLT3-ITD mutations are considered to confer a significantly poor outcome, these guidelines suggest that patients with these mutations should be considered for clinical trials if possible [5]. As for the ELN guidelines, these have recently undergone substantial revisions, the most significant of which includes categorization into only three risk groups based on genetics vs. four groups in the earlier classification. Importantly, with these revisions, AML with FLT3-ITD ${ }^{\text {high }}$ allelic ratio $(>0.5)$ in the absence of mutant nucleophosmin (NPM1) was moved into the high-risk group. Other significant changes include the addition of a new response category, "complete remission with no evidence of measurable residual disease," and the inclusion of additional gene mutations (RUNX1, ASXL1, and TP53) defining high risk. A new provisional response category, "progressive disease," was also added, particularly for use in clinical trials. The response category "stable disease," with the period of stable disease considered to be at least 3 months [4], was also added for clinical trial use.

Both the NCCN and ELN guidelines recommend the inclusion of FLT3 genetic testing in the diagnostic workup. More specifically, the NCCN guidelines recommend that FLT3 testing be performed at diagnosis in all patients with AML, in parallel with cytogenetic testing, to identify those who may benefit from targeted treatment options [5]. ELN recommends that, along with FLT3-ITD screening, mutantto-wild-type allelic ratio and tyrosine kinase domain
(TKD) mutations at codons D835 and I836 should be assessed.

The risk classification for NPM1 and FLT3-ITD genotypes in the 2017 ELN recommendations is presented in Table 2 [4] and was validated in a large retrospective analysis in patients with newly diagnosed AML and intermediate-risk cytogenetic abnormalities or normal karyotype [8]. A challenge with the ELN risk stratification is that it relies on FLT3-ITD allelic ratio data, which has yet to become part of the standard testing in clinical practice and is often unavailable to treating physicians. Additionally, while higher FLT3-ITD allelic ratios have been recognized for their association with poorer clinical outcomes [4], there is currently no internationally standardized methodology for determining these allelic ratios [9], further confounding the issue.

The observation that FLT3 mutations can evolve from diagnosis to relapse suggests that testing for FLT3-ITD mutations may be necessary at multiple time points throughout a patient's disease course to help guide the most appropriate therapeutic decisions. Because both the NCCN and ELN 2017 guidelines support prompt, comprehensive FLT3 testing for all patients with AML [4, 5], use of a rapid FLT3-ITD diagnostic assay has the potential to improve patient care by identifying patients with AML with a poor prognosis and allowing early intervention with FLT3-ITD targeted therapies [9-11]. However, there are several challenges. For example, interpretation of test results may be difficult due to variability in diagnostic accuracy, sensitivity, and qualitative vs. quantitative readouts of different FLT3 assays. Another source of variability stems from the timing of the testing and how patients are subsequently managed based on the physician's interpretation of the assay results. Additionally, FLT3-ITD allelic ratio has been 
Table 2 ELN 2017 AML risk stratification by genetics [4]

\begin{tabular}{|c|c|}
\hline Risk status & Genetic abnormality \\
\hline Favorable & $\begin{array}{l}\mathrm{t}(8 ; 21)(\mathrm{q} 22 ; \mathrm{q} 22.1) ; R U N X 1-R U N X 1 T 1 \\
\text { inv(16)(p13.1;q22) or } \mathrm{t}(16 ; 16)(\mathrm{p} 13.1 ; \mathrm{q} 22) ; C B F B- \\
M Y H 11 \\
\text { Mutated } N P M 1 \text { without } F L T 3-I T D \text { or with } F L T 3- \\
\text { ITD }{ }^{\text {low,a }} \\
\text { Biallelic mutated } C E B P A\end{array}$ \\
\hline Intermediate & $\begin{array}{l}\text { Mutated } N P M 1 \text { and } F L T 3-\text { ITD }^{\text {high,a }} \\
\text { Wild-type } N P M 1 \text { without } F L T 3 \text {-ITD or with FLT3- } \\
\text { ITD }^{\text {low,a }} \text { (without adverse-risk genetic lesions) } \\
\mathrm{t}(9 ; 11)(\mathrm{p} 21.3 ; \mathrm{q} 23.3) ; M L L T 3-K M T 2 A^{\mathrm{b}} \\
\text { Cytogenetic abnormalities not classified as favorable } \\
\text { or adverse }\end{array}$ \\
\hline Adverse & $\begin{array}{l}\mathrm{t}(6 ; 9)(\mathrm{p} 23 ; \mathrm{q} 34.1) ; D E K-N U P 214 \\
\mathrm{t}(\mathrm{v} ; 11 \mathrm{q} 23.3) ; K M T 2 A \text { rearranged } \\
\mathrm{t}(9 ; 22)(\mathrm{q} 34.1 ; \mathrm{q} 11.2) ; B C R-A B L 1 \\
\operatorname{inv}(3)(\mathrm{q} 21.3 ; \mathrm{q} 26.2) \text { or } \mathrm{t}(3 ; 3)(\mathrm{q} 21.3 ; \mathrm{q} 26.2) ; G A T A 2, \\
M E C O M(E V I 1) \\
-5 \text { or del }(5 \mathrm{q}) ;-7 ;-17 / \mathrm{abn}(17 \mathrm{p}) \\
\text { Complex karyotype }{ }^{\mathrm{c}} \text { monosomal karyotype }^{\mathrm{d}} \\
\text { Wild-type } N P M 1 \text { and } F L T 3-\mathrm{ITD}^{\text {high,a }} \\
\text { Mutated } R U N X 1^{\mathrm{e}} \\
\text { Mutated } A S X L 1^{\mathrm{e}} \\
\text { Mutated } T P 53^{\mathrm{f}}\end{array}$ \\
\hline
\end{tabular}

$A M L$ acute myeloid leukemia, CEBPA CCAAT/enhancer-binding protein alpha, ELN European LeukemiaNet, FLT3 FMS-like tyrosine kinase 3, ITD internal tandem duplication, NPM1 nucleophosmin, WT wild-type

Frequencies, response rates, and outcome measures should be reported by risk category, and, if sufficient numbers are available, by specific genetic lesions indicated

Prognostic impact of a marker is treatment dependent and may change with new therapies

aLow, low allelic ratio $(<0.5)$; high, high allelic ratio $(\geq 0.5)$. Semiquantitative assessment of FLT3-ITD allelic ratio (using DNA fragment analysis) is determined as the ratio of the area under the curve "FLT3-ITD" divided by the area under the curve "FLT3-wildtype." Recent studies indicate that AML with NPM1 mutation and FLT3-ITD low allelic ratio may have a more favorable prognosis and that patients should not routinely be assigned to allogeneic hematopoietic stem cell transplant

${ }^{\mathrm{b}}$ The presence of $\mathrm{t}(9 ; 11)(\mathrm{p} 21.3 ; \mathrm{q} 23.3)$ takes precedence over rare, concurrent adverse-risk gene mutations

${ }^{c}$ Three or more unrelated chromosomal abnormalities in the absence of one of the World Health Organization-designated recurring translocations or inversions, ie, $\mathrm{t}(8 ; 21)$, inv(16) or $\mathrm{t}(16 ; 16), \mathrm{t}(9 ; 11)$, $\mathrm{t}(\mathrm{v} ; 11)(\mathrm{v} ; \mathrm{q} 23.3), \mathrm{t}(6 ; 9), \operatorname{inv}(3)$ or $\mathrm{t}(3 ; 3)$, AML with $B C R-A B L 1$

${ }^{\mathrm{d}}$ Defined by the presence of one single monosomy (excluding loss of $\mathrm{X}$ or $\mathrm{Y}$ ) in association with $\geq 1$ additional monosomy or structural chromosomal abnormality (excluding core-binding factor AML)

${ }^{\mathrm{e}}$ These markers should not be used as an adverse prognostic marker if they co-occur with favorable-risk AML subtypes

${ }^{\mathrm{f}}$ TP53 mutations are significantly associated with AML with complex and monosomal karyotypes

used more often for research purposes than in real-world practice; as such, the clinical impact of FLT3-ITD allelic ratio has thus far been assessed retrospectively in patient data sets but not confirmed prospectively. Lastly, the lack of standardized laboratory reference values for FLT3-ITD allelic ratio is a further limitation. These observations highlight an acute need for an international standard for FLT3 mutational testing (Table 3), reporting, and interpretation.

Although routine testing for FLT3 mutations in patients with cytogenetically normal AML has been recommended by the ELN since 2010 [10], FLT3 testing is not always performed. The significantly higher rates of FLT3 testing at academic centers than at community sites suggests that there is a lack of awareness about the therapeutic implications of molecular testing in the community setting [12]. To validate this hypothesis, a large survey of physicians from the United States and Europe was conducted to assess their current practices in ordering tests for the diagnosis of acute leukemia. Only $51 \%$ of respondents indicated that they tested for FLT3-ITD in new AML referrals. Flow cytometry and karyotyping, on the other hand, were found to be routinely performed for the diagnosis of acute leukemia [13]. With increasing recognition of the importance of routine testing for FLT3 mutations in AML and the availability of FLT3 inhibitors, the frequency of FLT3 testing will likely increase in the future.

\section{FLT3 mutations in AML}

FLT3 is a transmembrane ligand-activated receptor tyrosine kinase that is normally expressed by hematopoietic stem or progenitor cells and plays an important role in the early stages of both myeloid and lymphoid lineage development [14]. An extracellular ligand (FLT3 ligand) binds and activates FLT3, promoting cell survival, proliferation, and differentiation through various signaling pathways, including PI3K, RAS, and STAT5 [14]. Mutations of FLT3 are found in approximately $30 \%$ of newly diagnosed AML cases and occur as either ITDs $(\approx 25 \%)$ or point mutations in the TKD (7-10\%) $[5,9,15,16]$. FLT3-ITD occurs in the form of a replicated sequence in the juxtamembrane domain and/or TKD1 of the FLT3 receptor and varies in location and length within these domains. Both FLT3-ITD and FLT3-TKD mutations constitutively activate FLT3 kinase activity, resulting in proliferation and survival of AML [14].

FLT3-ITD ${ }^{\text {high }}$ is a driver mutation that presents with a high leukemic burden, confers a poor prognosis, and has a significant negative impact on the management of patients with AML [1, 6, 9, 17-19]. Initial evidence of FLT3-ITD as a driver mutation came from comparative studies assessing the presence of this mutation in bone marrow (BM) samples at diagnosis and subsequently at relapse [20, 21]. A significant increase in the FLT3-ITD allelic ratio in relapsed AML indicates that a FLT3-ITD-mutated subclone present 
at diagnosis may possess a growth advantage and, through clonal expansion, become the dominant clone at relapse. Therefore, the FLT3-ITD mutation directly or indirectly confers a selective advantage to a clone in its microenvironment. Indeed, about $75 \%$ of patients with FLT3ITD-mutated AML at diagnosis continue to have the ITD mutation at relapse [22], suggesting that FLT3-ITD may function as the driver mutation responsible for progressing the disease into overt leukemia. Additionally, more recent evidence from a large study of the mutational landscape in adult AML identified FLT3 as one of the most commonly occurring mutations; specifically, FLT3-ITD was found to be one of the three most common drivers in patients with AML with intermediate-risk karyotypes (based on Medical Research Council classification) [23, 24], providing further support of its role as a driver mutation. More definitive evidence was observed with the first clinical use of potent FLT3 inhibitors, which resulted in the emergence of resistance-conferring point mutations [25], thus highlighting the function of FLT3-ITD mutations as drivers. In particular, the next-generation, highly specific FLT3 inhibitors -including gilteritinib, crenolanib, and quizartinibdemonstrated high single-agent activity, confirming the therapeutic potential of this approach [26-30]. Conversely, the first-generation multitargeted tyrosine kinase inhibitors (TKIs) that also inhibit FLT3 (e.g., midostaurin and sorafenib) initially demonstrated only modest single-agent activity in relapsed FLT3-ITD-mutated AML [31, 32], albeit with improved response rates and survival when combined with chemotherapy [33, 34].

\section{Prognostic impact of FLT3 mutations in newly diagnosed AML}

Patients with FLT3-ITD mutations tend to have a particularly unfavorable prognosis, with an increased risk of relapse and shorter overall survival (OS) compared with patients without the mutation $[35,36]$. A recent metaanalysis demonstrated that the presence of FLT3-ITD is associated with a poor prognosis in terms of OS and relapse-free survival (RFS; hazard ratios of 1.86 and 1.75, respectively) [37]. In contrast, the prognostic impact of FLT3-TKD mutations is not as well defined. For example, several studies have found weak associations between clinical outcomes and the presence of FLT3-TKD mutations, whereas at least one other, large study found no association with event-free survival (EFS) or OS [38].

Mutant-to-wild-type allelic ratio, insertion site, ITD length, karyotype, and the presence of a mutation in the $N P M 1$ gene appear to further influence the prognostic utility of FLT3-ITD in patients with newly diagnosed FLT3-ITDmutated AML. Higher allelic burden with the FLT3-ITD 
mutation has been specifically associated with worse outcomes in some studies but not in others [18, 39, 40]. For example, in a study evaluating the prognostic significance of FLT3-ITD in subgroups of patients with newly diagnosed FLT3-ITD-mutated AML, a threshold of mutant-to-wildtype ratio of $>0.78$ was significantly associated with shorter OS and disease-free survival (DFS) [18]. Similarly, in another study in patients with newly diagnosed FLT3-ITDmutated AML, a high allelic ratio $(\geq 0.51)$ and a FLT3-ITD insertion site in TKD1 predicted low complete remission (CR) rates and poor survival [39]. Notably, in both studies, patients were not treated with FLT3 inhibitors. In the RATIFY study, OS (not censored for transplant) was improved with midostaurin vs. placebo in groups with FLT3-ITD $^{\text {high }}$ and FLT3-ITD ${ }^{\text {low }}$ allelic burdens, suggesting that both patients with high and low mutant-to-wild-type ratio may benefit from the addition of midostaurin [41]. Conversely, in a large cohort of patients in Medical Research Council trials, Linch et al. [40] found that the risk of relapse did not correlate with the allelic ratio. Taken together, the interpretation of the data on allelic ratio remains controversial in this setting, and further studies are needed to better elucidate the prognostic impact of allelic burden so that treatment decisions based on it may be optimized. Other FLT3-ITD-related variables that may have prognostic significance in this setting are the base pair size of the FLT3-ITD mutation and its insertion site; e.g., increasing FLT3-ITD size has been shown to be associated with decreasing OS and RFS [42-44].

\section{Prognostic impact of FLT3 mutations in relapsed/refractory $A M L$}

An important concept in relapsed AML is that of clonal evolution, whereby mutations, such as FLT3-ITD mutations, that were not originally detectable at diagnosis can appear at relapse and may further affect prognosis [20, 21, 45-47]. In this setting (i.e., at relapse), AML is more oligoclonal, with leukemic clones harboring multiple adverserisk genetic mutations, and appears to be more dependent on, or "addicted" to, FLT3 signaling, at least in vitro [48]. In most patients with a FLT3-ITD mutation at diagnosis, the FLT3-ITD mutation is retained at relapse, with a higher allelic burden at relapse than at diagnosis [48]. However, other clonal possibilities may occur as the disease progresses from diagnosis to relapse. Pooled data from several studies have shown that nearly $20 \%$ of patients with AML have a newly detectable or lose a previously detectable FLT3-ITD or FLT3-TKD mutation at relapse [46]. More specifically, FLT3-ITD mutations are newly detected at relapse more often than FLT3-TKD mutations (8\% vs. $2 \%$ ), whereas previously detected FLT3-TKD mutations are lost at relapse more frequently than FLT3-ITD mutations (7\% vs. 4\%) [46]. This pattern is consistent with observations suggesting that AML harboring a FLT3-TKD mutation at diagnosis may be more chemosensitive than AML harboring a FLT3-ITD mutation [38, 46]. Although the mechanisms underlying alterations in FLT3 mutational status during clonal evolution remain unclear, it has been hypothesized that, in some cases, FLT3 mutations may simply be present at diagnosis at levels below the limits of detection of conventional assays. Therefore, clones with FLT3-ITD mutations, although undetectable at diagnosis, may eventually become dominant at relapse due to the survival advantage conferred by the mutation. This is especially true after the selective pressure of harsh chemotherapy.

Clonal evolution is particularly important because gaining FLT3-ITD mutations at relapse has been associated with shorter OS than maintaining WT FLT3 mutations [49]. FLT3-ITD mutations at relapse have also been shown to be an independent negative prognostic factor in patients in whom induction chemotherapy failed [50]. Moreover, Wattad and colleagues showed that patients with FLT3ITD-mutated AML on salvage therapy had a high risk of relapse even after a potentially curative allogeneic hematopoietic stem cell transplant (alloHSCT), with the FLT3ITD allelic ratio correlating directly with survival [50]-an observation that underscores patients' poor prognosis in this setting. Similar findings were observed in another study evaluating the effect of pretreatment characteristics on clinical outcomes, whereby patients with FLT3-ITD at relapse had a very low probability of achieving a second CR with standard intensive therapy and patients with a high FLT3-ITD allelic ratio continued to have a dismal prognosis even after alloHSCT [51]. Overall, as in the newly diagnosed treatment setting, the presence of a FLT3-ITD mutation in relapsed/refractory AML is associated with shorter duration of remission, increased risk of relapse, and decreased OS following standard-of-care therapy.

As the use of molecular data for predicting prognosis in AML becomes more common, one application of these data may be to define the role of HSCT in various prognostic groups. For example, Oran et al. recently showed that alloHSCT at first CR is associated with a prolonged RFS and OS that is independent of the FLT3-ITD allelic ratio and NPM1 mutation status in patients with a FLT3-ITD mutation [52]. Similarly, Ho et al. identified a clear EFS and OS benefit for alloHSCT vs. consolidation chemotherapy in patients identified as having high-risk FLT3-ITD-mutated AML and in patients with low-risk FLT3-ITD-mutated AML and WT NPM1 who received alloHCT in first remission; in patients with low-risk FLT3-ITD-mutated AML and NPM1 mutation a benefit from alloHSCT was not evident in terms of EFS and OS [53]. While some might interpret such studies to indicate that some subsets 
of patients with FLT3-ITD-mutated AML (defined by NPM1 mutation status and low allelic ratio) should receive consolidation chemotherapy in preference to alloHSCT, the lack of a standard method of determining the allelic ratio makes this problematic. Given that no studies we are aware of have found alloHSCT to be harmful in these patients, a safer course is simply to offer transplant to all patients with FLT3-ITD-mutated AML in first remission when feasible.

Of note, the clinical course of AML is also influenced by specific combinations of mutations rather than individual mutations, as demonstrated in a recent study assessing the driver landscape in AML through the identification of nonoverlapping subgroups of patients [24]. The design of this study allowed a full genomic classification and found, for example, that the NPM1 mutation carries a favorable prognosis only in the absence of a FLT3-ITD mutation (or FLT3-ITD with a low allelic ratio), whereas mutations in both ASXL1 and RUNX1 confer a poor prognosis, especially when they co-occur [54]. Collectively, although clinical outcomes in FLT3-ITD-mutated AML are complex and impacted by multiple factors, such as the patient's baseline characteristics and co-occurring mutations, there is robust evidence that FLT3-ITD is an important prognostic biomarker, as recognized in international guidelines (e.g., NCCN and ELN).

\section{FLT3 inhibitors}

Given the high frequency with which FLT3 mutations occur in AML, a number of TKIs are under development that disrupt the oncogenic signaling initiated by FLT3. In addition to a variety of improved treatment strategies in AML, the recognition that FLT3-ITD is an adverse prognostic marker, the integration of FLT3 inhibitors into the treatment algorithm, and the increased use of alloHSCT have led to improvements over the past 15 years in clinical outcomes in patients with FLT3-ITD-mutated AML. Notably, this trend was observed retrospectively in a singletertiary-center study evaluating differences in clinical outcomes in patients with newly diagnosed FLT3-ITD-mutated AML from 2000 to 2014, whereby a higher proportion of patients achieved CR in successive years and the corresponding median OS and median time to relapse increased significantly and incrementally over time with the introduction of alloHSCT and FLT3 inhibitors for the treatment of patients with FLT3-ITD mutations [55]. Overall, the use of FLT3 inhibitors, compared with historical outcomes prior to their emergence, has demonstrated a substantial clinical benefit in the relapsed/refractory AML setting and offers promising treatment strategies for patients with few options. For example, quizartinib, an oral, highly potent, and selective next-generation FLT3 inhibitor [56], significantly improved OS in a retrospective analysis in patients with FLT3-ITD-mutated AML who had relapsed after alloHSCT or after failure of second-line salvage chemotherapy compared with similar patients not treated with FLT3 inhibitors in the UK National Cancer Research Institute AML database (1988-2013) [57]. A summary of the first- and nextgeneration FLT3 inhibitors is presented in Table 4 and Fig. 1.

\section{First-generation TKIs}

Several multitargeted TKIs, such as lestaurtinib, sunitinib, sorafenib, and midostaurin, have activity against FLT3 and have been investigated in patients with FLT3-ITD-mutated AML [31, 58-61]. Early-phase studies evaluating these first-generation FLT3 inhibitors as monotherapy generally demonstrated limited antileukemic activity, mixed results when these agents were combined with chemotherapy, and increased toxicity in some cases [61, 62]. For example, lestaurtinib following salvage chemotherapy elicited no improvement in response rates or OS compared with salvage chemotherapy alone in patients with relapsed FLT3mutated AML [63], and combining lestaurtinib with intensive chemotherapy yielded no overall clinical benefit in patients with newly diagnosed FLT3-ITD-mutated AML [64]. Limited single-agent antileukemic activity was also shown in the phase 1 study of sunitinib, in which treatment of patients with refractory or resistant AML elicited only short-lived, partial responses [65]. Conversely, a phase 1/ 2 study evaluating sunitinib in combination with standard induction and cytarabine/daunorubicin consolidation therapy demonstrated that $50 \%$ of patients with FLT3-ITD mutations and 38\% with FLT3-TKD mutations achieved CR [61]. In this study, two patients receiving sunitinib 25 mg daily continuously from day 1 onward (dose 1) and one patient receiving sunitinib $25 \mathrm{mg}$ daily only on days $1-7$ of each cycle (dose -1$)$ experienced dose-limiting toxicities (i.e., prolonged time to recovery of peripheral blood counts and hand-foot syndrome at dose 1 and neutropenia at dose -1) that necessitated dose reductions [61].

As for sorafenib, similarly limited single-agent antileukemic activity was observed in a phase 1 study evaluating sorafenib monotherapy in patients with relapsed/refractory FLT3-mutated AML (the study included two patients with other acute leukemias and a minority of patients without FLT3 mutations [22\%]), in which only $10 \%$ of patients achieved CR or CR with incomplete platelet recovery [32]. Conversely, a phase 2 trial in younger patients (aged $\leq 60$ years) with previously untreated AML found that the addition of sorafenib to standard-of-care chemotherapy significantly prolonged EFS and RFS but not OS compared with placebo. However, this treatment regimen was also associated with increased toxicity [62]. Moreover, in an 
Table 4 First- and next-generation FLT3 inhibitors [79, 81, 88, 99, 100]

Key pathways targeted (in addition to Developmental phase FLT3)

First-generation FLT3 inhibitors

\begin{tabular}{|c|c|c|c|}
\hline Sunitinib & VEGFR2, PDGFR $\beta$, KIT, RET & Phase 2 & $\begin{array}{l}\text { Decreased appetite, } \\
\text { headache, GI symptoms }\end{array}$ \\
\hline Sorafenib & $\begin{array}{l}\text { RAF, VEGFR1/2/3, PDGFR } \beta \text {, KIT, } \\
\text { RET }\end{array}$ & Phase 3 & Skin rash, fatigue, diarrhea \\
\hline Midostaurin & $\begin{array}{l}\text { PKC, SYK, FLK- } 1, \text { AKT, PKA, KIT, } \\
\text { FGR, SRC, PDGFR } \alpha / \beta, \text { VEGFR } 1 / 2\end{array}$ & $\begin{array}{l}\text { Approved for the treatment of newly diagnosed FLT3- } \\
\text { mutated AML in combination with chemotherapy }\end{array}$ & $\begin{array}{l}\text { Fever, flu-like symptoms, } \\
\text { mouth sores, unusual } \\
\text { bleeding or bruising }\end{array}$ \\
\hline Lestaurtinib & JAK2/3, TrkA/B/C & Phase 2 & $\begin{array}{l}\text { Infections, sepsis, } \\
\text { myocardial infarction }\end{array}$ \\
\hline Ponatinib & $\begin{array}{l}\text { LYN, ABL, PDGFR } \alpha, \text { VEGFR2, } \\
\text { FGFR1, SRC, KIT, TEK, RET }\end{array}$ & Phase 2 & Pancreatitis \\
\hline Tandutinib & KIT, PDGFR $\beta$ & Withdrawn & Muscle weakness \\
\hline KW-2449 & $\mathrm{ABL}$, aurora kinase & Withdrawn & NA \\
\hline \multicolumn{4}{|c|}{ Next-generation FLT3 inhibitors } \\
\hline Crenolanib & PDGFR $\beta$ & Phase 3 & $\begin{array}{l}\text { Nausea, vomiting, transaminitis, } \\
\text { fluid retention }\end{array}$ \\
\hline Quizartinib & KIT, PDGFR & Phase 3 & $\begin{array}{l}\text { QTcF prolongation (especially } \\
\text { at higher doses) }\end{array}$ \\
\hline Gilteritinib & LTK, ALK, AXL & Phase 3 & $\begin{array}{l}\text { Diarrhea, fatigue, high liver } \\
\text { function tests }\end{array}$ \\
\hline
\end{tabular}

FGFR fibroblast growth factor receptor, FLT3 FMS-like tyrosine kinase 3, GI gastrointestinal, JAK Janus kinase, NA not applicable, PDGFR platelet-derived growth factor receptor, $P K$ protein kinase, $V E G F R$ vascular endothelial growth factor receptor

Fig. 1 Type I FLT3: inhibitors bind the FLT3 receptor in the active conformation, either near the activation loop or the ATPbinding pocket, and are active against ITD and TKD mutations. Type II FLT3 inhibitors bind the FLT3 receptor in the inactive conformation in a region adjacent to the ATP-binding domain. As a result of this binding affinity, type II FLT3 inhibitors prevent activity of ITD mutations but do not target TKD mutations [81]. FLT3, FMS-like tyrosine kinase; ITD, internal tandem duplication; JMD, juxtamembrane domain; TK, tyrosine kinase; TKD, tyrosine kinase domain

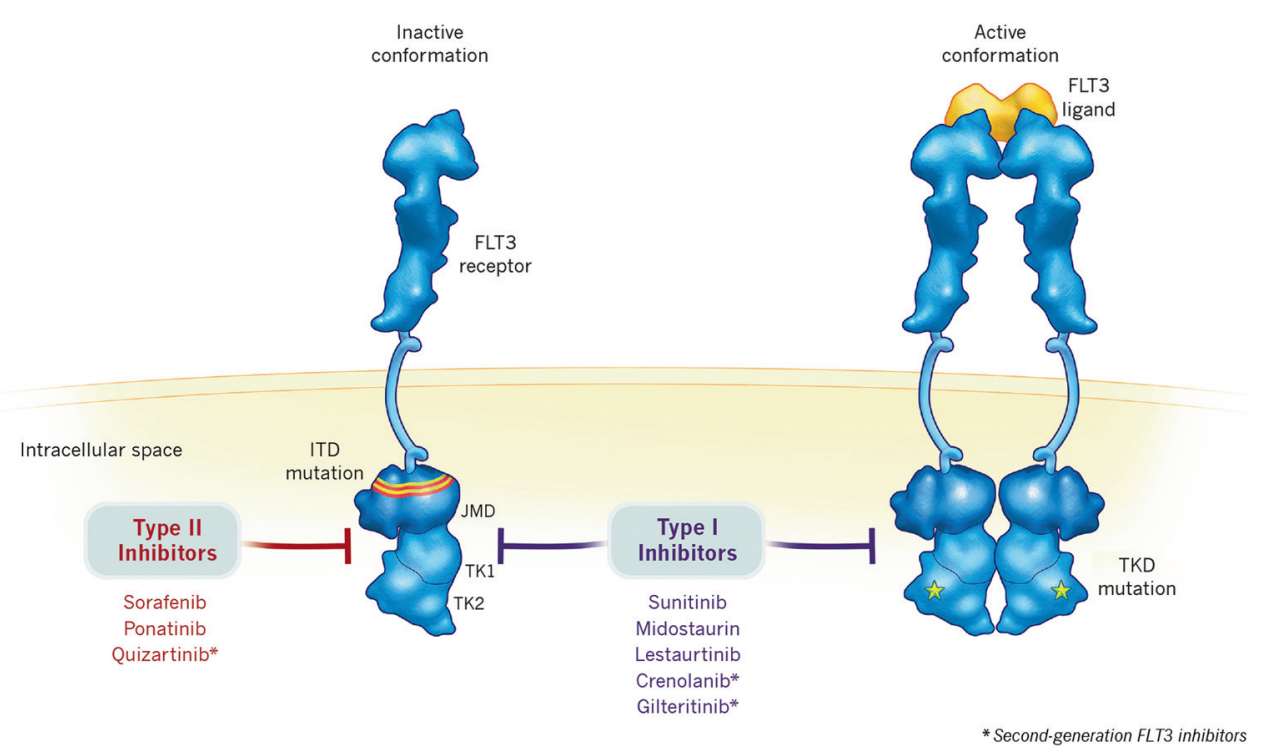

exploratory subgroup analysis, no EFS benefit was observed with sorafenib in the small group of patients with FLT3-ITD mutations; however, patients with non-FLT3 mutations had significantly improved EFS and RFS [62]. In another study, the combination of sorafenib with standard 7 +3 chemotherapy in elderly patients (aged $>60$ years) with
Main toxicities fluid retention

QTcF prolongation (especially

Diarrhea, fatigue, high liver function tests 
AML, the combination of sorafenib, cytarabine, and idarubicin resulted in high response rates at a median follow-up of 52 months. CR/CR with incomplete platelet recovery rates of 95\% were achieved in patients with FLT3-ITD mutations. DFS and OS of 13.8 and 29 months, respectively, were also improved compared with historical outcomes in these patients [34]. As yet another example, encouraging response rates $(46 \%$ overall response rate [ORR]) were achieved in a nonrandomized study of azacytidine plus sorafenib in patients with relapsed/refractory AML, including 93\% with FLT3-ITD mutations [67]. Finally, in the post-alloHSCT setting, phase 1 data suggest that sorafenib has good tolerability at twice-daily doses between 200 and $400 \mathrm{mg}$ and a 1-year progression-free survival of $85 \%$ in all patients was reported, including several patients who underwent transplant beyond their first CR [68].

The use of single-agent midostaurin in patients with relapsed/refractory FLT3-mutated AML was similarly shown to have limited antileukemic activity, although it was generally well tolerated [31, 69]. Therefore, midostaurin was further investigated in combination with standard $7+3$ chemotherapy in patients with newly diagnosed AML (aged 18-59 years) with FLT3 mutations and was shown to significantly improve EFS (hazard ratio $=0.78 ; P=0.002$ ) and OS (hazard ratio $=0.78 ; P=0.009$ ) compared with 7 +3 chemotherapy alone [70]. On the basis of the outcomes from this pivotal phase 3 trial (RATIFY), midostaurin (in combination with standard cytarabine and daunorubicin induction and cytarabine consolidation therapy) was approved by the US Food and Drug Administration (FDA) in April 2017 for the treatment of adult patients with newly diagnosed FLT3-mutated AML as detected by an FDAapproved test $[71,72]$. In Europe, marketing authorization for midostaurin was granted by the European Commission on September 20, 2017, and includes an indication for single-agent maintenance therapy for adult patients in CR following induction/consolidation with $7+3$ chemotherapy and midostaurin [73]. Despite that patients $>60$ years were not enrolled in RATIFY, there are no restrictions for midostaurin use in patients aged 65 and over, other than caution based on a patient's eligibility for concomitant chemotherapy and potential for comorbidities (i.e., greater frequency of concomitant disease or other drug therapy) [74]. Importantly, in patients receiving an HSCT, midostaurin should be discontinued prior to HSCT. Midostaurin was not approved by the FDA as maintenance therapy beyond induction and consolidation [71].

Data from the RATIFY trial showed that midostaurin consistently improved OS in all FLT3 mutation subtypes (i.e., TKD, ITD low allelic ratio, and ITD high allelic ratio), with some differential effects observed among the four major genotypes studied (i.e., a significant OS and EFS benefit with midostaurin was observed only in the NPM1WT/FLT3-ITD-high subgroup) and by gender (i.e., no significant OS benefit in men with FLT3-TKD mutations or women with FLT3-ITD mutations) [70, 75]. These results suggest that the prognostic impact and predictive value of individual mutations may be significantly impacted by concurrent mutations. However, given that patients across all FLT3 subtypes-regardless of FLT3-ITD allelic ratiobenefited and that midostaurin inhibits multiple kinases, it is possible that midostaurin's favorable effects may not stem purely from FLT3 inhibition but could be attributable at least in part to inhibition of other oncogenic pathways. Of note, $23 \%$ of the study population in the RATIFY trial had a FLT3-TKD mutation, which is a significantly larger percentage than the previously reported incidence of TKD mutations in the general AML population [41]. Patients with FLT3-TKD-mutated AML have lower white blood cell counts and generally have less-aggressive disease than patients with FLT3-ITD-mutated AML, allowing additional time for workup and screening for trials. This may have biased outcomes in this trial in favor of patients with a FLT3-TKD mutation, a phenomenon that was repeated in a similar trial of chemotherapy plus lestaurtinib [64]. Moreover, patients in the RATIFY trial were younger (median age $=47.9$ years) than typical patients with newly diagnosed AML, highlighting the need for additional data for midostaurin in elderly fit and unfit patients [76, 77].

Although midostaurin is now approved, the debate is still ongoing as to how it impacts OS as well as its role in maintenance therapy. To this end, an exploratory analysis including CRs according to the protocol specifications (CRs up to day 60) showed that midostaurin improved the CR rate significantly after induction therapy when expanded CRs were counted $(P=0.04)$ [41]. Midostaurin was most effective in patients who received an alloHSCT in first CR, with a nonsignificant, in-trend better survival $(P=0.07)$ and a significantly lower cumulative incidence of relapse $(P=0.02)$ in all patients achieving a $\mathrm{CR}$ after induction therapy [70]. In contrast, patients who received chemotherapy without alloHSCT as consolidation therapy had a comparable cumulative incidence of relapse rate regardless of whether they received midostaurin. In a post hoc analysis, there appeared to be no benefit of midostaurin (DFS, $P=0.38$; OS, $P=0.86$ ) in patients who proceeded to midostaurin maintenance therapy [78].

Several phase 2 studies to further evaluate midostaurin in patients with newly diagnosed FLT3-ITD-mutated AML are ongoing. The first (AMLSG 16-10), a single-arm, phase 2 trial (NCT01477606; ongoing, but closed for recruitment) [79], is assessing the addition of midostaurin to chemotherapy during induction and consolidation as well as single-agent midostaurin maintenance for a maximum follow-up of 1 year in patients aged 18 to 70 years with 
newly diagnosed FLT3-ITD-positive AML. The second (RADIUS) is an ongoing, randomized, open-label, phase 2 trial evaluating the addition of midostaurin to standard of care vs. standard of care alone in the post-transplant setting in patients with FLT3-mutated AML who underwent alloHSCT and have not relapsed (NCT01883362). Preliminary results from both phase 2 trials suggest that these approaches are feasible [76, 80]. Of note, following early observations that patients with FLT3-WT experienced blast reductions with midostaurin, a phase 3 study in FLT3-WT AML has recently initiated (NCT003512197) [69].

\section{Next-generation TKIs}

Overall, these multitargeted TKIs lack specificity for the mutated FLT3-ITD, which may explain their transient antileukemic activity, particularly when used as monotherapy in patients with relapsed disease [69], and may contribute to adverse effects from inhibition of multiple other kinases. To overcome these hurdles, several nextgeneration FLT3 inhibitors are under clinical investigation in AML, including gilteritinib, crenolanib, and quizartinib. These next-generation inhibitors have greater specificity for FLT3 and higher potency (logarithmically lower halfmaximal inhibitory concentration) than multitargeted TKIs [58]. Gilteritinib and crenolanib are type I inhibitors that target both the inactive and active conformational states of the FLT3 kinase domain, whereas quizartinib is a type II inhibitor that is specific for the inactive conformation [81].

Next-generation FLT3 inhibitors have shown promising single-agent antileukemic activity in early clinical trials. For example, in a first-in-human, phase $1 / 2$, dose-escalation and -expansion trial examining the effect of gilteritinib monotherapy in 252 patients aged $\geq 18$ years with relapsed/ refractory AML, gilteritinib treatment elicited a 40\% ORR (including composite $\mathrm{CR}$ [CRc] and partial remission [PR]) in efficacy-evaluable patients $(n=249)$. The CRc rate was $30 \%$, with the majority of responders having CR with incomplete blood count recovery (CRi) [29]. Response rates were considerably lower in patients with WT FLT3 (ORR, 12\%; CRc, 9\%), whereas patients with FLT3 mutations had an ORR of $49 \%$ and a CRc rate of $37 \%$. Response rates increased with higher doses of gilteritinib; patients receiving $\geq 80 \mathrm{mg} /$ day of gilteritinib achieved an ORR of $52 \%$ and a $\mathrm{CRc}$ rate of $41 \%$. The median duration of response, defined as the time from the date of first CRc or PR until the date of relapse, was 17 weeks. Interestingly, the CRc rate with gilteritinib was $26 \%$ in patients treated with prior FLT3 inhibitors [29].

Additionally, single-agent crenolanib has demonstrated activity in the relapsed/refractory setting in two phase 2 studies in patients with relapsed/refractory FLT3-mutated AML. In the first, a single-center, phase 2 trial in 38 patients, crenolanib elicited a CRi rate of $23 \%$ in FLT3 inhibitor-naive patients and a CRi rate of $5 \%$ in those previously treated with FLT3 inhibitors. The median OS in the two groups was 55 and 13 weeks, respectively [30]. Results from the second, larger phase 2 study $(N=69)$ revealed a CRi rate of $39 \%$ and a PR rate of $11 \%$ in patients with relapsed FLT3-mutated AML who had not received prior FLT3 inhibitors and a CRi + PR rate of $28 \%$ in patients who had received prior FLT3 inhibitors. The median OS was 33.4 weeks in patients with relapsed/refractory FLT3mutated AML who were TKI naive; OS was longest in those with FLT3-ITD mutations (34 weeks) and patients aged $<60$ years (33.4 weeks) [26].

Lastly, the type II inhibitor quizartinib—an oral, highly potent, and selective next-generation FLT3 inhibitor [56, 82, 83] - has been evaluated in QuANTUM-R, a global, randomized, open-label, phase 3 study (NCT02039726) examining the effect of quizartinib monotherapy vs. salvage chemotherapy (randomized 2:1) on OS in 367 patients with FLT3-ITD-mutated AML who are refractory to or have relapsed after first-line therapy. Other than rare cases of grade $\geq 3$ QTcF prolongation with quizartinib, adverse event (AE) rates were comparable between the two arms.

Quizartinib has also been shown to be highly active in phase 2 trials, resulting in a high proportion of responders across many patient types with relapsed/refractory disease $[27,28]$. For example, in a large phase 2 study (two cohorts; 333 patients), single-agent quizartinib (90 or $135 \mathrm{mg} /$ day) resulted in CRc rates of 46 to $56 \%$ and ORRs of $74-77 \%$, improved OS in responders compared with nonresponders, and was generally well tolerated, with a manageable safety profile [27]. In patients aged $\geq 60$ years with FLT3-ITDmutated AML relapsed within 1 year of initial remission or refractory to first-line chemotherapy (cohort $1 ; n=157$ ), the CRc rate was $56 \%$ and the ORR was $77 \%$. Median duration of CRc was 12.1 weeks in FLT3-ITD-positive patients and 16.4 weeks in FLT3-ITD-negative patients. Median OS was 25.4 and 19.1 weeks in FLT3-ITD-positive and FLT3-ITDnegative patients, respectively. Similarly, in patients aged $\geq 18$ years with AML relapsed/refractory to second-line salvage chemotherapy or relapsed after HSCT (cohort 2; $n=176$ ), the CRc rate was $46 \%$ and the ORR was $74 \%$. The median duration of CRc was 10.6 weeks in FLT3-ITDpositive patients and 7.0 weeks in FLT3-ITD-negative patients. In this cohort, the median OS was 24.0 and 25.1 weeks in FLT3-ITD-positive and FLT3-ITD-negative patients, respectively. Toxicity was consistent with phase 1 data and was generally well managed by dose interruptions and/or reductions [27].

Consistent with observations from the large, two-cohort, phase 2 trial, a phase $2 b$ study evaluating the effects of quizartinib at lower doses (starting doses of 30 or $60 \mathrm{mg}$ / day) in a similar patient population (i.e., relapsed/refractory 
FLT3-ITD-mutated AML; $N=76$ ) showed strong singleagent clinical activity, with an overall CRc of $47 \%$ [28]. The $60-\mathrm{mg} /$ day dose (vs. $30 \mathrm{mg} /$ day) was associated with a higher ORR (71\%), median OS (27.3 weeks), and bridge to transplant rate $(42 \%)$, reinforcing the promising antileukemic activity of single-agent quizartinib observed in earlier studies and warranting further investigation of the $60-\mathrm{mg}$ dosing regimen [28]. Additionally, initial experience with quizartinib in combination with chemotherapy in younger [84] and older patients with newly diagnosed AML [85] and in combination with azacytidine or low-dose cytarabine in older patients with newly diagnosed FLT3-ITD-mutated AML and those with FLT3-ITD-mutated AML in first relapse (including myelodysplastic syndromes and chronic myelomonocytic leukemia) [86] indicated that the combinations were feasible and appeared effective in both younger and older patients. Quizartinib also seems to be well tolerated as a single-agent therapy following alloHSCT in patients with FLT3-ITD-mutated AML who are in remission [87]. Together, these findings suggest that targeting the FLT3-ITD driver mutation with a highly potent and selective FLT3 inhibitor is a promising clinical strategy to help improve clinical outcomes in patients with very few options.

From a safety perspective, AEs associated with nextgeneration FLT3 inhibitors are generally manageable. Common treatment-related AEs with gilteritinib were diarrhea $(37 \%)$, anemia (34\%), fatigue (33\%), increased aspartate aminotransferase $(26 \%)$, and increased alanine aminotransferase (19\%) [29]. Crenolanib was associated with grade 3 gastrointestinal toxicities and mild or moderate nausea/ vomiting, transaminitis, and fluid retention [26, 30]. Treatment-related treatment-emergent AEs occurring in patients treated with higher doses of quizartinib (i.e., $\geq 90 \mathrm{mg} /$ day) were primarily myelosuppression and grade $\geq 3 \mathrm{QTcF}$ prolongation (10\%), which was reversible and successfully managed by treatment interruptions or dose reductions. Lower doses of quizartinib were associated with significantly lower rates of QTcF prolongation $(<5 \%$ grade $\geq 3 \mathrm{QTcF}$ prolongation) while maintaining high levels of efficacy.

\section{Ongoing trials of FLT3 inhibitors}

New TKIs, such as ponatinib and FLX925, are being evaluated in phase 1 studies [88, 89]. However, none of these agents has demonstrated appreciable single-agent activity thus far. Given the favorable response and safety profiles of next-generation FLT3 inhibitors, several phase 3 trials are currently underway with gilteritinib, crenolanib, and quizartinib in a variety of settings. Two phase 3 studies are evaluating the effects of gilteritinib as maintenance therapy following alloHSCT (NCT02997202) and following induction/consolidation therapy (NCT02927262), respectively. Crenolanib is being evaluated in two phase 3 studies, including in combination with chemotherapy in patients with relapsed/refractory AML and FLT3 mutations (NCT02298166) and in a randomized, head-to-head study of crenolanib vs. midostaurin in combination with standard first-line treatment for AML (NCT03258931). In addition to QuANTUM-R (described above), quizartinib is being evaluated in QuANTUM-First, a global, randomized, double-blind, placebo-controlled, phase 3 study (NCT02668653) examining the effect of quizartinib plus standard induction and consolidation chemotherapy followed by single-agent quizartinib on EFS in patients with newly diagnosed primary FLT3-ITD-mutated AML. The results from these studies may provide additional treatment options to a particularly vulnerable patient population.

\section{Mechanisms of resistance to FLT3 inhibitors}

Despite significant progress in the development of newer FLT3 inhibitors with greater potency and specificity, emergence of resistance poses a significant challenge [81]. Both inherent and acquired mechanisms contribute to drug resistance. WT FLT3 is sensitive to FLT3 ligand and is relatively resistant to FLT3 inhibitors; therefore, the presence of WT FLT3 in most patients with FLT3-ITD mutations may contribute to resistance to FLT3 inhibitors. High levels of FLT3 ligand found in the BM microenvironment during induction as well as consolidation therapy can lead to persistent activity of the FLT3/MAPK pathway and provide survival signals to leukemic blasts, even in the presence of FLT3 inhibitors at levels that produce effective inhibition of FLT3 kinase activity in vitro $[90,91]$. Persistent activation of pathways downstream of FLT3, such as MAPK and STAT5, has also been shown to contribute to inherent resistance to FLT3 inhibitors [92, 93]. Suboptimal efficacy of FLT3 inhibitors similarly may arise due to inadequate drug concentrations in the plasma, possibly due to rapid metabolism in the liver by cytochrome P450 A4 (CYP3A4) enzymes [94]. Finally, microenvironmental factors may influence the sensitivity of leukemic cells to TKIs. Preclinical studies have demonstrated that CYP3A4 expressed in BM stromal cells enhances drug metabolization and contributes to BM microenvironment-mediated FLT3 TKI resistance [95]. Acquired FLT3 point mutations such as TKD mutations may arise from therapy with type I and II FLT3 inhibitors and mediate resistance $[25,96]$.

Secondary mutation-driven acquired resistance represents a common but complex mechanism underlying resistance to targeted therapies. Different FLT3 kinase inhibitors generate distinct, nonoverlapping secondary FLT3 
resistance mutations [97]. Additional resistance mechanisms include acquisition of other gene mutations and activation of alternative signaling pathways during treatment with FLT3 inhibitors [92]. Alterations in key signaling pathways in FLT3 TKI-resistant cell lines and primary samples reveal other forms of resistance, including activation of PI3K/AKT and/or RAS/MEK/MAPK pathways as well as continued expression of genes involved in FLT3mediated cellular transformation [92].

An important strategy to overcome resistance to chemotherapy in many tumor types has been the use of combination regimens [81]. To this end, several ongoing studies are evaluating the utility of combining different agents that inhibit key signaling pathways through different modes of action or by using two or more agents that target different leukemic cell survival signaling pathways. Planned and ongoing studies investigating this clinical strategy include combinations of FLT3 inhibitors with approved antileukemic therapies, such as hypomethylating agents, low-dose cytarabine, CPX-351, and investigational agents (e.g., MDM2, BCL-2, IDH1/2, bromodomain, MEK, and CYP3A4 inhibitors). Combination therapies not only may improve response rates but also may produce more durable remissions in patients with FLT3-mutated AML.

Acknowledgments Financial support for medical editorial assistance, copyright permission, and publication costs was provided by Daiichi Sankyo, Inc., a member of the Daiichi Sankyo Group. Medical writing assistance was provided by Jamie King, PhD, of SciMentum, Inc, a Nucleus Global Company.

Author contributions The authors met the criteria for authorship as recommended by the International Committee of Medical Journal Editors (ICMJE). The authors were fully responsible for all content and editorial decisions, were involved at all stages of manuscript development, and approved the final version that reflects the authors' interpretations and conclusions.

\section{Compliance with ethical standards}

Conflict of interest ND has received research funding from Daiichi Sankyo, Bristol-Myers Squibb, Pfizer, Karyopharm, Sevier, Genentech, and ImmunoGen and has served in a consulting or advisory role for Daiichi Sankyo, Bristol-Myers Squibb, Pfizer, Novartis, Celgene, AbbVie, and Agios. RFS has received honoraria from Daiichi Sankyo, Novartis, Pfizer, Janssen, and Arog; has served in a consulting or advisory role for Daiichi Sankyo, Novartis, and Pfizer; and has received research funding from Pfizer, AstraZeneca, PharmaMar, and Novartis. NHR has received honoraria from and has served in an advisory role for Jazz, Teva, Pfizer, and Daiichi Sankyo. MJL has received honoraria from Daiichi Sankyo, Novartis, and Agios; has served in a consulting or advisory role for Daiichi Sankyo, Novartis, and Agios; and has received research funding from Astellas and Novartis.

Publisher's note: Springer Nature remains neutral with regard to jurisdictional claims in published maps and institutional affiliations.
Open Access This article is licensed under a Creative Commons Attribution 4.0 International License, which permits use, sharing, adaptation, distribution and reproduction in any medium or format, as long as you give appropriate credit to the original author(s) and the source, provide a link to the Creative Commons license, and indicate if changes were made. The images or other third party material in this article are included in the article's Creative Commons license, unless indicated otherwise in a credit line to the material. If material is not included in the article's Creative Commons license and your intended use is not permitted by statutory regulation or exceeds the permitted use, you will need to obtain permission directly from the copyright holder. To view a copy of this license, visit http://creativecommons. org/licenses/by/4.0/.

\section{References}

1. Ding L, Ley TJ, Larson DE, Miller CA, Koboldt DC, Welch JS, et al. Clonal evolution in relapsed acute myeloid leukaemia revealed by whole-genome sequencing. Nature. 2012;481:50610.

2. Döhner H, Weisdorf DJ, Bloomfield CD. Acute myeloid leukemia. N Engl J Med. 2015;373:1136-52.

3. Walter MJ, Payton JE, Ries RE, Shannon WD, Deshmukh H, Zhao $\mathrm{Y}$, et al. Acquired copy number alterations in adult acute myeloid leukemia genomes. Proc Natl Acad Sci USA. 2009;106:12950-5.

4. Döhner H, Estey E, Grimwade D, Amadori S, Appelbaum FR, Buchner T, et al. Diagnosis and management of AML in adults: 2017 ELN recommendations from an international expert panel. Blood. 2017;129:424-47.

5. O'Donnell MR, Tallman MS, Abboud CN, Altman JK, Appelbaum FR, Arber DA, et al. Acute myeloid leukemia, version 3.2017, NCCN Clinical Practice Guidelines in Oncology. J Natl Compr Canc Netw. 2017;15:926-57.

6. Grimwade D, Mrozek K. Diagnostic and prognostic value of cytogenetics in acute myeloid leukemia. Hematol Oncol Clin North Am. 2011;25:61. vii

7. Arber DA, Orazi A, Hasserjian R, Thiele J, Borowitz MJ, Le Beau MM. et al. The 2016 revision to the World Health Organization classification of myeloid neoplasms and acute leukemia. Blood. 2016;127:2391-405.

8. Schetelig J, Rollig C, Kayser S, Stoelzel F, Schaefer-Eckart K, Haenel M, et al. Validation of the ELN 2017 Classification for AML with intermediate risk cytogenetics with or without NPM1mutations and high or low Ratio FLT3-ITDs. Blood. 2017;130:2694.

9. Levis M. FLT3 mutations in acute myeloid leukemia: what is the best approach in 2013? Hematol Am Soc Hematol Educ Program. 2013;2013:220-6.

10. Döhner H, Estey EH, Amadori S, Appelbaum FR, Buchner T, Burnett AK, et al. Diagnosis and management of acute myeloid leukemia in adults: recommendations from an international expert panel, on behalf of the European LeukemiaNet. Blood. 2010;115:453-74.

11. Grunwald MR, Tseng LH, Lin MT, Pratz KW, Eshleman JR, Levis MJ, et al. Improved FLT3 internal tandem duplication PCR assay predicts outcome after allogeneic transplant for acute myeloid leukemia. Biol Blood Marrow Transplant. 2014;20:1989-95.

12. Lin TL, Williams T, He J, Aljitawi OS, Ganguly S, Abhyankar S, et al. Rates of complete diagnostic testing for patients with acute myeloid leukemia. Cancer Med. 2015;4:519-22. 
13. George TI, Tworek JA, Thomas NE, Fatheree LA, Souers RJ, Nakhleh RE, et al. Evaluation of testing of acute leukemia samples: survey result from the College of American Pathologists. Arch Pathol Lab Med. 2017;141:1101-6.

14. Grafone T, Palmisano M, Nicci C, Storti S. An overview on the role of FLT3-tyrosine kinase receptor in acute myeloid leukemia: biology and treatment. Oncol Rev. 2012;6:e8.

15. Kottaridis PD, Gale RE, Linch DC. Flt3 mutations and leukaemia. Br J Haematol. 2003;122:523-38.

16. Nagel G, Weber D, Fromm E, Erhardt S, Lubbert M, Fiedler W, et al. Epidemiological, genetic, and clinical characterization by age of newly diagnosed acute myeloid leukemia based on an academic population-based registry study (AMLSG BiO). Ann Hematol. 2017;96:1993-2003.

17. Whitman SP, Archer KJ, Feng L, Baldus C, Becknell B, Carlson $\mathrm{BD}$, et al. Absence of the wild-type allele predicts poor prognosis in adult de novo acute myeloid leukemia with normal cytogenetics and the internal tandem duplication of FLT3: a Cancer and Leukemia Group B study. Cancer Res. 2001;61:7233-9.

18. Thiede C, Steudel C, Mohr B, Schaich M, Schakel U, Platzbecker U, et al. Analysis of FLT3-activating mutations in 979 patients with acute myelogenous leukemia: association with FAB subtypes and identification of subgroups with poor prognosis. Blood. 2002;99:4326-35.

19. Khaled S, Al Malki M, Marcucci G. Acute myeloid leukemia: biologic, prognostic, and therapeutic insights. Oncology (Williston Park). 2016;30:318-29.

20. Kottaridis PD, Gale RE, Langabeer SE, Frew ME, Bowen DT, Linch DC. Studies of FLT3 mutations in paired presentation and relapse samples from patients with acute myeloid leukemia: implications for the role of FLT3 mutations in leukemogenesis, minimal residual disease detection, and possible therapy with FLT3 inhibitors. Blood. 2002;100:2393-8.

21. Shih LY, Huang CF, Wu JH, Lin TL, Dunn P, Wang PN, et al. Internal tandem duplication of FLT3 in relapsed acute myeloid leukemia: a comparative analysis of bone marrow samples from 108 adult patients at diagnosis and relapse. Blood. 2002;100:2387-92.

22. Kronke J, Bullinger L, Teleanu V, Tschurtz F, Gaidzik VI, Kuhn MW, et al. Clonal evolution in relapsed NPM1-mutated acute myeloid leukemia. Blood. 2013;122:100-8.

23. Metzeler KH, Herold T, Rothenberg-Thurley M, Amler S, Sauerland MC, Gorlich D, et al. Spectrum and prognostic relevance of driver gene mutations in acute myeloid leukemia. Blood. 2016;128:686-98.

24. Papaemmanuil E, Gerstung M, Bullinger L, Gaidzik VI, Paschka $\mathrm{P}$, Roberts ND, et al. Genomic classification and prognosis in acute myeloid leukemia. N Engl J Med. 2016;374:2209-21.

25. Smith CC, Wang Q, Chin CS, Salerno S, Damon LE, Levis MJ, et al. Validation of ITD mutations in FLT3 as a therapeutic target in human acute myeloid leukaemia. Nature. 2012;485: 260-3.

26. Cortes JE, Kantarjian HM, Kadia TM, Borthakur G, Konopleva M, Garcia-Manero G. et al. Crenolanib besylate, a type I panFLT3 inhibitor, to demonstrate clinical activity in multiply relapsed FLT3-ITD and D835 AML. J Clin Oncol. 2016; 34:7008

27. Cortes J, Perl AE, Döhner H, Kantarjian H, Martinelli G, Kovacsovics T, et al. Quizartinib, an FLT3 inhibitor, as monotherapy in patients with relapsed or refractory acute myeloid leukaemia: an open-label, multicentre, single-arm, phase 2 trial. Lancet Oncol. 2018;19:889-903.

28. Cortes JE, Tallman MS, Schiller GJ, Trone D, Gammon G, Goldberg SL, et al. Phase $2 b$ study of two dosing regimens of quizartinib monotherapy in FLT3-ITD mutated, relapsed or refractory AML. Blood. 2018;132:598-607.
29. Perl AE, Altman JK, Cortes J, Smith C, Litzow M, Baer MR, et al. Selective inhibition of FLT3 by gilteritinib in relapsed or refractory acute myeloid leukaemia: a multicentre, first-inhuman, open-label, phase 1-2 study. Lancet Oncol. 2017;18:1061-75.

30. Randhawa JK, Kantarjian HM, Borthakur G, Thompson PA, Konopleva M, Daver N, et al. Results of a phase II study of crenolanib in relapsed/refractory acute myeloid leukemia patients (Pts) with activating FLT3 mutations. Blood. 2014;124:389.

31. Stone RM, DeAngelo DJ, Klimek V, Galinsky I, Estey E, Nimer $\mathrm{SD}$, et al. Patients with acute myeloid leukemia and an activating mutation in FLT3 respond to a small-molecule FLT3 tyrosine kinase inhibitor, PKC412. Blood. 2005;105:54-60.

32. Borthakur G, Kantarjian H, Ravandi F, Zhang W, Konopleva M, Wright JJ, et al. Phase I study of sorafenib in patients with refractory or relapsed acute leukemias. Haematologica. 2011;96:62-8.

33. Stone RM, Fischer T, Paquette R, Schiller G, Schiffer CA, Ehninger G, et al. Phase IB study of the FLT3 kinase inhibitor midostaurin with chemotherapy in younger newly diagnosed adult patients with acute myeloid leukemia. Leukemia. 2012;26:2061-8.

34. Ravandi F, Arana YiC, Cortes JE, Levis M, Faderl S, GarciaManero G, et al. Final report of phase II study of sorafenib, cytarabine and idarubicin for initial therapy in younger patients with acute myeloid leukemia. Leukemia. 2014;28:1543-5.

35. Kiyoi H, Naoe T, Nakano Y, Yokota S, Minami S, Miyawaki S, et al. Prognostic implication of FLT3 and N-RAS gene mutations in acute myeloid leukemia. Blood. 1999;93:3074-80.

36. Kottaridis PD, Gale RE, Frew ME, Harrison G, Langabeer SE, Belton AA, et al. The presence of a FLT3 internal tandem duplication in patients with acute myeloid leukemia (AML) adds important prognostic information to cytogenetic risk group and response to the first cycle of chemotherapy: analysis of 854 patients from the United Kingdom Medical Research Council AML 10 and 12 trials. Blood. 2001;98:1752-9.

37. Port M, Bottcher M, Thol F, Ganser A, Schlenk R, Wasem J, et al. Prognostic significance of FLT3 internal tandem duplication, nucleophosmin 1, and CEBPA gene mutations for acute myeloid leukemia patients with normal karyotype and younger than 60 years: a systematic review and meta-analysis. Ann Hematol. 2014;93:1279-86.

38. Bacher U, Haferlach C, Kern W, Haferlach T, Schnittger S. Prognostic relevance of FLT3-TKD mutations in AML: the combination matters-an analysis of 3082 patients. Blood. 2008;111:2527-37.

39. Schlenk RF, Kayser S, Bullinger L, Kobbe G, Casper J, Ringhoffer M, et al. Differential impact of allelic ratio and insertion site in FLT3-ITD-positive AML with respect to allogeneic transplantation. Blood. 2014;124:3441-9.

40. Linch DC, Hills RK, Burnett AK, Khwaja A, Gale RE. Impact of FLT3(ITD) mutant allele level on relapse risk in intermediaterisk acute myeloid leukemia. Blood. 2014;124:273-6.

41. Stone RM, Mandrekar SJ, Sanford BL, Laumann K, Geyer S, Bloomfield CD, et al. Midostaurin plus chemotherapy for acute myeloid leukemia with a FLT3 mutation. N Engl J Med. 2017;377:454-64.

42. Stirewalt DL, Kopecky KJ, Meshinchi S, Engel JH, PogosovaAgadjanyan EL, Linsley J, et al. Size of FLT3 internal tandem duplication has prognostic significance in patients with acute myeloid leukemia. Blood. 2006;107:3724-6.

43. Kayser S, Schlenk RF, Londono MC, Breitenbuecher F, Wittke $\mathrm{K}, \mathrm{Du} \mathrm{J}$, et al. Insertion of FLT3 internal tandem duplication in the tyrosine kinase domain-1 is associated with resistance to chemotherapy and inferior outcome. Blood. 2009;114:2386-92.

44. Liu SB, Dong HJ, Bao XB, Qiu QC, Li HZ, Shen HJ, et al. Impact of FLT3-ITD length on prognosis of acute myeloid leukemia. Haematologica. 2019;104:e9-e12. 
45. Cloos J, Goemans BF, Hess CJ, van Oostveen JW, Waisfisz Q, Corthals S, et al. Stability and prognostic influence of FLT3 mutations in paired initial and relapsed AML samples. Leukemia. 2006;20:1217-20.

46. McCormick SR, McCormick MJ, Grutkoski PS, Ducker GS, Banerji N, Higgins RR, et al. FLT3 mutations at diagnosis and relapse in acute myeloid leukemia: cytogenetic and pathologic correlations, including cuplike blast morphology. Arch Pathol Lab Med. 2010;134:1143-51.

47. Nazha A, Cortes J, Faderl S, Pierce S, Daver N, Kadia T, et al. Activating internal tandem duplication mutations of the fms-like tyrosine kinase-3 (FLT3-ITD) at complete response and relapse in patients with acute myeloid leukemia. Haematologica. 2012;97:1242-5.

48. Pratz KW, Sato T, Murphy KM, Stine A, Rajkhowa T, Levis M. FLT3-mutant allelic burden and clinical status are predictive of response to FLT3 inhibitors in AML. Blood. 2010;115:1425-32.

49. Warren M, Luthra R, Yin CC, Ravandi F, Cortes JE, Kantarjian HM, et al. Clinical impact of change of FLT3 mutation status in acute myeloid leukemia patients. Mod Pathol. 2012;25:1405-12.

50. Wattad M, Weber D, Döhner K, Krauter J, Gaidzik VI, Paschka $\mathrm{P}$, et al. Impact of salvage regimens on response and overall survival in acute myeloid leukemia with induction failure. Leukemia. 2017;31:1306-13.

51. Schlenk RF, Frech P, Weber D, Brossart P, Horst HA, Kraemer $\mathrm{D}$, et al. Impact of pretreatment characteristics and salvage strategy on outcome in patients with relapsed acute myeloid leukemia. Leukemia. 2017;31:1217-20.

52. Oran B, Cortes J, Beitinjaneh A, Chen HC, de Lima M, Patel K, et al. Allogeneic transplantation in first remission improves outcomes irrespective of FLT3-ITD allelic ratio in FLT3-ITDpositive acute myelogenous leukemia. Biol Blood Marrow Transplant. 2016;22:1218-26.

53. Ho AD, Schetelig J, Bochtler T, Schaich M, Schafer-Eckart K, Hanel M, et al. Allogeneic stem cell transplantation improves survival in patients with acute myeloid leukemia characterized by a high allelic ratio of mutant FLT3-ITD. Biol Blood Marrow Transplant. 2016;22:462-9.

54. Gaidzik VI, Teleanu V, Papaemmanuil E, Weber D, Paschka P, Hahn J, et al. RUNX1 mutations in acute myeloid leukemia are associated with distinct clinico-pathologic and genetic features. Leukemia. 2016;30:2160-8.

55. Badar T, Kantarjian HM, Nogueras-Gonzalez GM, Borthakur G, Garcia Manero G, Andreeff M, et al. Improvement in clinical outcome of FLT3 ITD mutated acute myeloid leukemia patients over the last one and a half decade. Am J Hematol. 2015;90: 1065-70.

56. Zarrinkar PP, Gunawardane RN, Cramer MD, Gardner MF, Brigham D, Belli B, et al. AC220 is a uniquely potent and selective inhibitor of FLT3 for the treatment of acute myeloid leukemia (AML). Blood. 2009;114:2984-92.

57. Hills RK, Gammon G, Trone D, Burnett AK. Quizartinib significantly improves overall survival in FLT3-ITD positive AML patients relapsed after stem cell transplantation or after failure of salvage chemotherapy: a comparison with historical AML database (UK NCRI data). Blood. 2015;126:2557.

58. Wander SA, Levis MJ, Fathi AT. The evolving role of FLT3 inhibitors in acute myeloid leukemia: quizartinib and beyond. Ther Adv Hematol. 2014;5:65-77.

59. Smith BD, Levis M, Beran M, Giles F, Kantarjian H, Berg K, et al. Single-agent CEP-701, a novel FLT3 inhibitor, shows biologic and clinical activity in patients with relapsed or refractory acute myeloid leukemia. Blood. 2004;103: 3669-76.

60. Pratz KW, Cortes J, Roboz GJ, Rao N, Arowojolu O, Stine A, et al. A pharmacodynamic study of the FLT3 inhibitor KW-2449 yields insight into the basis for clinical response. Blood. 2009;113:3938-46.

61. Fiedler W, Kayser S, Kebenko M, Janning M, Krauter J, Schittenhelm M, et al. A phase I/II study of sunitinib and intensive chemotherapy in patients over 60 years of age with acute myeloid leukaemia and activating FLT3 mutations. Br J Haematol. 2015;169:694-700.

62. Rollig C, Serve H, Huttmann A, Noppeney R, Muller-Tidow C, Krug U, et al. Addition of sorafenib versus placebo to standard therapy in patients aged 60 years or younger with newly diagnosed acute myeloid leukaemia (SORAML): a multicentre, phase 2, randomised controlled trial. Lancet Oncol. 2015;16:1691-9.

63. Levis M, Ravandi F, Wang ES, Baer MR, Perl A, Coutre S, et al. Results from a randomized trial of salvage chemotherapy followed by lestaurtinib for patients with FLT3 mutant AML in first relapse. Blood. 2011;117:3294-301.

64. Knapper S, Russell N, Gilkes A, Hills RK, Gale RE, Cavenagh $\mathrm{JD}$, et al. A randomized assessment of adding the kinase inhibitor lestaurtinib to first-line chemotherapy for FLT3-mutated AML. Blood. 2017;129:1143-54.

65. Fiedler W, Serve H, Döhner H, Schwittay M, Ottmann OG, O'Farrell AM, et al. A phase 1 study of SU11248 in the treatment of patients with refractory or resistant acute myeloid leukemia (AML) or not amenable to conventional therapy for the disease. Blood. 2005;105:986-93.

66. Serve H, Krug U, Wagner R, Sauerland MC, Heinecke A, Brunnberg U, et al. Sorafenib in combination with intensive chemotherapy in elderly patients with acute myeloid leukemia: results from a randomized, placebo-controlled trial. J Clin Oncol. 2013;31:3110-8.

67. Ravandi F, Alattar ML, Grunwald MR, Rudek MA, Rajkhowa T, Richie MA, et al. Phase 2 study of azacytidine plus sorafenib in patients with acute myeloid leukemia and FLT-3 internal tandem duplication mutation. Blood. 2013;121:4655-62.

68. Chen YB, Li S, Lane AA, Connolly C, Del Rio C, Valles B, et al. Phase I trial of maintenance sorafenib after allogeneic hematopoietic stem cell transplantation for fms-like tyrosine kinase 3 internal tandem duplication acute myeloid leukemia. Biol Blood Marrow Transplant. 2014;20:2042-8.

69. Fischer T, Stone RM, Deangelo DJ, Galinsky I, Estey E, Lanza C, et al. Phase IIB trial of oral midostaurin (PKC412), the FMSlike tyrosine kinase 3 receptor (FLT3) and multi-targeted kinase inhibitor, in patients with acute myeloid leukemia and high-risk myelodysplastic syndrome with either wild-type or mutated FLT3. J Clin Oncol. 2010;28:4339-45.

70. Stone RM, Mandrekar SJ, Sanford BL, Laumann K, Geyer SM, Bloomfield $\mathrm{CD}$, et al. The addition of midostaurin to standard chemotherapy decreases cumulative incidence of relapse (CIR) in the international prospective randomized, placebo-controlled, double-blind trial (CALGB 10603 / RATIFY [Alliance]) for newly diagnosed acute myeloid leukemia (AML) patients with FLT3 mutations. Blood. 2017;130:2580.

71. Rydapt PI. 2018. https://www.accessdata.fda.gov/drugsatfda docs/label/2017/207997s000lbl.pdf; Accessed 2018.

72. Novartis Pharmaceuticals Corporation. Novartis receives FDA approval for Rydapt ${ }^{\circledR}$ in newly diagnosed FLT3-mutated acute myeloid leukemia (AML) and three types of systemic mastocytosis (SM). 2018. https://www.novartis.com/news/media-relea ses/novartis-receives-fda-approval-rydaptr-newly-diagnosed-flt3mutated-acute. Accessed 2018.

73. Rydapt SMC. 2017. http://www.ema.europa.eu/docs/en_GB/ document_library/EPAR_-_Product_Information/human/ 004095/WC500237581.pdf. Accessed 2018.

74. Rydapt SMC. 2018. https://www.ema.europa.eu/documents/ product-information/rydapt-epar-product-information_en.pdf; Accessed 2018. 
75. Döhner K, Thiede C, Larson RA, Prior TW, Marcucci G, Jones $\mathrm{D}$, et al. Prognostic impact of NPM1/FLT3-ITD genotypes from randomized patients with acute myeloid leukemia (AML) treated within the international RATIFY Study. Blood. 2017;130:467.

76. Schlenk R, Döhner K, Salih H, Kündgen A, Fiedler W, Salwender $\mathrm{H}$, et al. Midostaurin in combination with intensive induction and as single agent maintenance therapy after consolidation therapy with allogeneic hematopoietic stem cell transplantation or high-dose cytarabine (NCT01477606). Blood. 2015;126:322.

77. Schlenk RF, Fiedler W, Salih HR, Wulf G, Thol F, Kündgen A, et al. Impact of age and midostaurin-dose on response and outcome in acute myeloid leukemia with FLT3-ITD: interimanalyses of the AMLSG 16-10 Trial. Blood. 2016;128:449.

78. Larson RA, Mandrekar SJ, Sanford BL, Laumann K, Geyer SM, Bloomfield $\mathrm{CD}$, et al. An analysis of maintenance therapy and post-midostaurin outcomes in the international prospective randomized, placebo-controlled, double-blind trial (CALGB 10603/ RATIFY [Alliance]) for newly diagnosed acute myeloid leukemia (AML) patients with FLT3 mutations. Blood. 2017;130:145.

79. National Institutes of Health. ClinicalTrials.gov. https://clinica ltrials.gov/. Accessed 2018.

80. Maziarz RT, Patnaik MM, Scott BL, Mohan SR, Deol A, Rowley SD, et al. RADIUS: a phase 2, randomized trial of standard of care (SOC) with or without midostaurin to prevent relapse following allogeneic hematopoietic stem cell transplant (alloHSCT) in patients (pts) with FLT3-Itd-mutated acute myeloid leukemia (AML). Blood. 2016;128:2248.

81. Daver N, Cortes J, Ravandi F, Patel KP, Burger JA, Konopleva $\mathrm{M}$, et al. Secondary mutations as mediators of resistance to targeted therapy in leukemia. Blood. 2015;125:3236-45.

82. Chao Q, Sprankle KG, Grotzfeld RM, Lai AG, Carter TA, Velasco AM, et al. Identification of N-(5-tert-butyl-isoxazol-3yl)-N'-\{4-[7-(2-morpholin-4-yl-ethoxy)imidazo[2,1-b][1, 3]benzothiazol-2-yl]phenyl \}urea dihydrochloride (AC220), a uniquely potent, selective, and efficacious FMS-like tyrosine kinase-3 (FLT3) inhibitor. J Med Chem. 2009;52:7808-16.

83. Gunawardane RN, Nepomuceno RR, Rooks AM, Hunt JP, Ricono JM, Belli B, et al. Transient exposure to quizartinib mediates sustained inhibition of FLT3 signaling while specifically inducing apoptosis in FLT3-activated leukemia cells. Mol Cancer Ther. 2013;12:438-47.

84. Altman JK, Foran JM, Pratz KW, Trone D, Cortes JE, Tallman MS. Phase 1 study of quizartinib in combination with induction and consolidation chemotherapy in patients with newly diagnosed acute myeloid leukemia. Am J Hematol. 2018;93:213-21.

85. Bowen D, Russell N, Knapper S, Milligan D, Hunter AE, Khwaja A, et al. AC220 (quizartinib) can be safely combined with conventional chemotherapy in older patients with newly diagnosed acute myeloid leukaemia: experience from the AML18 Pilot Trial. Blood. 2013;122:622.

86. Swaminathan M, Kantarjian HM, Daver N, Borthakur G, Ohanian M, Kadia $\mathrm{T}$, et al. The combination of quizartinib with azacitidine or low dose cytarabine is highly active in patients (pts) with FLT3-ITD mutated myeloid leukemias: interim report of a phase I/II trial. Blood. 2017;130:723.
87. Sandmaier BM, Khaled S, Oran B, Gammon G, Trone D, Frankfurt O. Results of a phase 1 study of quizartinib as maintenance therapy in subjects with acute myeloid leukemia in remission following allogeneic hematopoietic stem cell transplant. Am J Hematol. 2018;93:222-31.

88. Shah NP, Talpaz M, Deininger MW, Mauro MJ, Flinn IW, Bixby D, et al. Ponatinib in patients with refractory acute myeloid leukaemia: findings from a phase 1 study. Br J Haematol. 2013;162:548-52.

89. Daver N, Pollyea DA, Rizzieri DA, Palmer J, Rampal RK, Dinner S, et al. A phase I study of FLX925, a dual FLT3 and CDK4/6 inhibitor in patients with relapsed or refractory acute myeloid leukemia (AML). Blood. 2017;130:1343.

90. Sato T, Yang X, Knapper S, White P, Smith BD, Galkin S, et al. FLT3 ligand impedes the efficacy of FLT3 inhibitors in vitro and in vivo. Blood. 2011;117:3286-93.

91. Yang X, Sexauer A, Levis M. Bone marrow stroma-mediated resistance to FLT3 inhibitors in FLT3-ITD AML is mediated by persistent activation of extracellular regulated kinase. Br J Haematol. 2014;164:61-72.

92. Piloto O, Wright M, Brown P, Kim KT, Levis M, Small D. Prolonged exposure to FLT3 inhibitors leads to resistance via activation of parallel signaling pathways. Blood. 2007;109: 1643-52.

93. Traer E, Martinez J, Javidi-Sharifi N, Agarwal A, Dunlap J, English I, et al. FGF2 from marrow microenvironment promotes resistance to FLT3 inhibitors in acute myeloid leukemia. Cancer Res. 2016;76:6471-82.

94. Dutreix C, Munarini F, Lorenzo S, Roesel J, Wang Y. Investigation into CYP3A4-mediated drug-drug interactions on midostaurin in healthy volunteers. Cancer Chemother Pharmacol. 2013;72:1223-34.

95. Chang Y, Hernandez D, Ghiaur G, Levis MJ, Jones RJ. Bone marrow stroma protects FLT3 acute myeloid leukemia (AML) through CYP3A4-mediated drug metabolization of FLT3 tyrosine kinase inhibitors (TKIs). Blood. 2017;130:2519.

96. Heidel F, Solem FK, Breitenbuecher F, Lipka DB, Kasper S, Thiede $\mathrm{MH}$, et al. Clinical resistance to the kinase inhibitor PKC412 in acute myeloid leukemia by mutation of Asn-676 in the FLT3 tyrosine kinase domain. Blood. 2006;107: 293-300.

97. von Bubnoff N, Engh RA, Aberg E, Sanger J, Peschel C, Duyster J. FMS-like tyrosine kinase 3-internal tandem duplication tyrosine kinase inhibitors display a nonoverlapping profile of resistance mutations in vitro. Cancer Res. 2009;69: 3032-41.

98. Patnaik MM. The importance of FLT3 mutational analysis in acute myeloid leukemia. Leuk Lymphoma. 2018;59:2273-86.

99. Kampa-Schittenhelm KM, Heinrich MC, Akmut F, Döhner H, Döhner K, Schittenhelm MM. Quizartinib (AC220) is a potent second generation class III tyrosine kinase inhibitor that displays a distinct inhibition profile against mutant-FLT3, -PDGFRA and -KIT isoforms. Mol Cancer. 2013;12:19.

100. Lee LY, Hernandez D, Rajkhowa T, Smith SC, Raman JR, Nguyen B, et al. Preclinical studies of gilteritinib, a nextgeneration FLT3 inhibitor. Blood. 2017;129:257-60. 